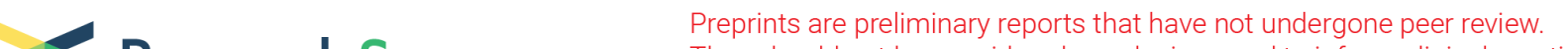 $\begin{array}{ll}\text { Research Square } & \begin{array}{l}\text { They should not be considered conclusive, used to inform clinical practice, } \\ \text { or referenced by the media as validated information. }\end{array}\end{array}$
}

\section{Factors Associated With Non-Compliance to Diabetes Medication in a Rapidly Urbanizing Region in Ghana: A Mixed-Methods Study.}

Dorice A. Berkoh ( $\sim$ dorice.berkoh@uenr.edu.gh )

Dept. of Basic and Applied Biology, University of Energy and Natural Resources

\section{William B. K. A. Owiredu}

Dept. of Molecular Medicine, School of Medicine and Dentistry, Kwame Nkrumah University of Science and Technology, Kumasi-Ghana

\section{Samuel F. Gyasi}

Dept. of Basic and Applied Biology, University of Energy and Natural Resources, Sunyani-Ghana

\section{Emmanuel T. Donkoh}

Dept. of Basic and Applied Biology, University of Energy and Natural Resources, Sunyani-Ghana.

\section{Robert Ngala}

Dept. of Molecular Medicine, School of Medicine and Dentistry, Kwame Nkrumah University of Science and Technology, Kumasi-Ghana

\section{Research Article}

Keywords: Type 2 diabetes, anti-diabetic medication, adherence, compliance, Ghana, Sunyani

Posted Date: February 28th, 2022

DOI: https://doi.org/10.21203/rs.3.rs-1403407/v1

License: @ (i) This work is licensed under a Creative Commons Attribution 4.0 International License.

Read Full License

Version of Record: A version of this preprint was published at Porto Biomedical Journal on May 1st, 2022. See the published version at https://doi.org/10.1097/j.pbj.0000000000000148. 


\section{Abstract \\ Background}

Failure to achieve adequate glycaemic control can lead to debilitating complications for diabetics. Strict compliance to prescribed diet, lifestyle and medication can prevent complications.

\section{Methods}

In order to examine factors accompanying non-compliance behaviour to diabetes medication in a rapidly urbanizing region of Ghana, a mixed approach was adopted. Study subjects (N: 160, mean age: 58.3) were interviewed at the Diabetic Clinic of the Brong-Ahafo Regional Hospital, Sunyani. Compliance to diabetes treatment was evaluated with an adapted Morisky Medication Adherence Scale (MMAS). Faceto-face interviews of 20 subjects allowed for more personalized exploration of psychosocial aspects of non-compliance. The interviews were audio recorded, transcribed verbatim, and coded using the Nvivo software. Qualitative data was processed and subjected to inductive thematic analysis.

\section{Results}

Majority of study participants reported an optimum $(n=121,75.6 \%)$ level of compliance to diabetes medication, although some also reported poor compliance $(n=39,24.4 \%)$. Qualitative responses received during interviews suggest that poor compliance may be attributable to misconceptions about religious beliefs and practices. Psychosocial factors relating to felt stress, the inevitability of fate and compliance fatigue were also discovered to undermine compliance. Non-compliance behaviour was also explained by socioeconomic status and barriers to health-seeking behaviour.

\section{Conclusion}

Reported medication compliance was among the highest in out-patient settings in Ghana. However, contextual determinants of non-compliance have to be addressed. Efforts to improve compliance to diabetic medication could benefit from interventions that address superstition, target psychological aspects of chronic disease management and remove operational barriers to healthcare delivery such as transportation costs and long waiting times.

\section{Introduction}

According to the International Diabetes Federation (IDF), there are over 463 million people living with diabetes worldwide. Africa has recorded 19 million cases across the region and this is expected to increase by $143 \%$ by the year $2045(1,2)$. Diabetes was scarcely a public health concern in sub-Saharan Africa, however, a number of factors have led to a surge in the number of patients attending routine 
diabetic clinics in Ghana $(1,3)$. Under the Ghana Health Service, district-level health facilities are mandated to organize separate diabetic clinics to manage patients. Management for type 2 diabetes includes early medication involving one or more oral hypoglycaemic drugs and lifestyle modification where patients are encouraged to maintain a healthy diet and follow a regular exercise plan $(2,4)$.

The available evidence suggests that complying with recommended lifestyle and medication modifications significantly influences the treatment and care of diabetes (5). However, compliance challenges are very common among patients; making it almost impossible to achieve proper glycaemic control $(6,7)$. The failure to achieve adequate glycaemic control can lead to debilitating complications that impair the quality of life of diabetic individuals $(8,9)$ and higher cost burden on the healthcare system (10).

Adherence or compliance to medication refers to "the extent to which a patient takes medication as prescribed by a healthcare provider" $(2,6,7,10)$. Medication compliance has conventionally been measured using pill counts and standardized questionnaires (11-13). The Morisky Medication Adherence Scale (MMAS) is one of the most popular instruments among researchers owing to its low cost and time expenditure (14). Non-compliance to diabetes medication is still an unresolved problem (5) in Ghana. Several reasons are given for non-compliance to medications. Some of these reasons include high cost of medicines, side effects, increased number and dosage of medicines, psychological effects, difficulty in remembering dosages and religious beliefs (15). Other explanations given for non-compliance are reported to be associated with psychosocial, cultural and socioeconomic factors as well as lack of satisfactory provision of effective guidance (16). The present study examines factors related to noncompliance behaviour of patients receiving treatment at a diabetes clinic in the rapidly urbanizing Bono Region of Ghana.

\section{Methodology}

\section{Study design and setting}

A mixed approach was selected to explore the contextual factors underpinning non-compliance to diabetic medication in the Diabetic Clinic (Clinic 5) of the Sunyani Regional Hospital in Ghana. The clinic, which is run by a medical team comprising specialist physicians, registered nurses, pharmacists and nutritionists saw 243 new and 3,490 old clients in 2019 (17).

\section{Sample size}

The required sample size was calculated by StatCalc application of Epilnfo 3.5.3. In a previous study, the prevalence of medication compliance among a cross-section of diabetes patients was $38.5 \%$ (18). After correcting for population size, a sample of 142 participants was adequate to estimate the prevalence of medication compliance with precision $(d=0.07)$ at $95 \%$ confidence level. After an initial piloting of study protocol, 165 diabetic patients were recruited. Out of this number five (5) questionnaires were excluded 
for incompleteness, refusal to provide consent after explaining study procedure, and language barrier leaving a total of 160 for analyses.

\section{Participants and recruitment}

Individuals diagnosed with diabetes aged 18 years and/or above and receiving treatment at the Diabetic Clinic (Clinic 5) of the Brong-Ahafo Regional Hospital in Sunyani $(N=160)$, Ghana were eligible to participate. Diabetic status was defined for patients who had been diagnosed with diabetes for one year or more and/or taking one or more oral hypoglycaemic drugs. Drug history was verified from patient folders.

\section{Data collection and ethical considerations}

Qualitative and quantitative data were collected using a semi-structured interview guide (S1 File). The interview guide, which was in English was translated by language experts and rendered in local dialect when needed. From July 2019 to November, 2019, information was collected about socio-demographics, family history, medication history as well as compliance. Compliance to medication was measured using the MMAS (Table 3) (14). The interview guide was pre-tested in the clinic prior to subject recruitment. Data collected during the pre-testing phase of the work was used to test the reliability of the instrument for measuring compliance to diabetic medication in the clinic setting. An alpha value of 0.79 was deemed proof of sufficient reliability after excluding the penultimate question to give a 7-item adapted instrument. The instrument was checked for validity by experienced academic supervisors and an expert in medication compliance research and necessary amendments were made. 
Table 3

Summary of responses to MMAS and categories of medication compliance

Item

Yes

$\mathrm{N} \quad \%^{2}$

$36 \quad 22.5$

1. Do you sometimes forget to take your medicine?

$30 \quad 18.8$

2. Over the past two weeks, were there any days when you did not take your medicine?

3. Have you ever cut back or stopped taking your medication without telling your doctor because you felt worse when you took it?

4. When you travel or leave home, do you sometimes forget to bring along your medications?

5. Did you take your medicine yesterday?

6. When you feel like your condition is under control, do you sometimes stop taking your medicine?

1

0.06

7. How often do you forget to take all your medications?

26

$16.3^{\#}$

\section{Categories of Medication Compliance}

Low compliance

$39 \quad 24.4$

Medium compliance

$121 \quad 75.6$

High compliance

0

0.0

Low compliance: a score of $>2$; Medium compliance: a score of 1 or 2: High compliance: a score of 0 . \# This response includes responses such as "once in a while", "sometimes", "usually" and "all the time". Medium to High levels of medication compliance are considered to be adequate for optimum glycaemic control (14).

One-on-one interviews lasting for about 30-45 minutes with probing queries were conducted at the clinic site. Participants were contacted before the interview through a telephone call to schedule their clinical appointment and explain study procedures to them. Data saturation occurred after 16 subjects and no new information emerged during a further 4 interviews.

\section{Ethical approval}

(KHRCIEC/2018-22) was obtained from the Kintampo Health Research Centre (KHRC) Institutional Ethics Committee with additional approval from the Diabetes clinic (Clinic 5) of the Brong-Ahafo Regional Hospital. Study participants were adequately informed of the purpose, nature, procedures, risks and hazards of the study. All participants signed informed consent forms according to the recommendations of the local ethics review committees that gave approval and were assured confidentiality and anonymity of details 


\section{Data Analysis}

Quantitative data were presented as count (percentage) and continuous variables as mean \pm standard deviation. Qualitative data from face-to-face interviews were recorded as .amr files and converted to .mp3 format, transcribed verbatim, and coded using the Nvivo software and analysed using the procedure of inductive thematic analysis (19). Synthesized claims were shared with five (5) randomly selected representative subjects of the study to establish congruity of primary findings with their views and experiences.

\section{Results}

Study participants were mostly middle-aged clients (mean age: $58.3, S D=10.7$ ), had been living with diabetes for almost a decade (mean since diagnosis: 8.8 years, $S D=5.7$ ) and generally overweight (mean BMI: $26.5, S D=4.7)$. A larger percentage of study participants were married $(n=88,55.0 \%)$, self-employed $(n=84,52.5 \%)$ or had a minimum of senior high school education $(n=80,50.0 \%)$ (Table 1$)$. According to clinic records, most participants under review were being managed for type 2 diabetes $(n=150,93.8 \%)$ (Table 2). 
Table 1

Demographic characteristics of study participants

\section{Variables}

$\mathbf{N}\left(\%^{1}\right)$

Age (years \pm SD)

Time since diagnosis (years \pm SD)

BMI Categories

Underweight

Normal

Overweight

Obese

$\mathrm{BMI}($ mean $\pm \mathrm{SD})$

Marital Status

Single

Married

Educational Level

Primary or below

High School

Tertiary

Occupational Status

Unemployment

Self-Employment

Employee

Pensioner

${ }^{1}$ Column proportions. Underweight: BMI is less than 18.5. Normal weight: $\mathrm{BMI}$ is 18.5 to 24.9.

Overweight: $\mathrm{BMI}$ is 25 to 29.9. Obese: $\mathrm{BMI}$ is 30 or more.
Total

$58.3 \pm 10.7$

$8.8 \pm 5.7$

$5(3.1)$

$59(36.9)$

$63(39.4)$

$31(19.4)$

$26.5 \pm 4.7$

$72(45.0)$

88 (55.0)

54 (33.8)

$80(50.0)$

26 (16.2)

23 (14.4)

84 (52.5)

12 (7.5)

41 (25.6) 
Table 2

Clinical characteristics of study participants

\section{Variables}

Diabetes status

Type 1

Type 2

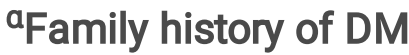

Yes

No

Don't know

${ }^{\beta}$ Time since diagnosis (yrs.)

$1-3$

$4-10$

$>10$

\section{Smoking history}

Yes

No

\section{Alcohol history}

Yes

No

${ }^{1}$ Column proportions. ' 'Yes' indicates that at least one known family member of the participant has been diagnosed with diabetes. ${ }^{\beta}$ Indicates the number of years a participant has been living with a diagnosis of diabetes.

\section{Total}

$\mathbf{N}\left(\%^{1}\right)$

$10(6.2)$

$150(93.8)$

$95(59.4)$

$44(27.5)$

$21(13.1)$

$27(16.9)$

$88(55.0)$

$45(28.1)$

$4(2.5)$

$156(97.5)$

$41(25.6)$

$119(74.4)$

Medication compliance was measured on an a modified MMAS with three outcomes: low (a score of $>2$ ), medium (a score of 1 or 2) and high (a score of 0 ) medication compliance based on a set of seven questions. Low $(n=39,24.4 \%)$ and medium $(n=121,75.6 \%)$ compliance was observed for participants based on the MMAS. None of the participants reported high medication compliance (Table 3).

\section{Factors associated with noncompliance to anti-diabetic medication}


Qualitative responses from patients suggested that the observed compliance to medication could be as a result of entrenched religious misconceptions regarding health and superstition. Psychosocial factors related to stress, fate, perception of health, compliance fatigue, socioeconomic factors and barriers to health-seeking behaviour were also among reasons given for non-compliance. Social support, however, for patients was reported to be adequate.

\section{Barriers related to religious beliefs and practices Belief in faith healing}

Religion is an integral aspect of social life in Ghana. All participants interviewed held on to some form of religious worldview. A number of study participants cited potential conflict between medical care and superstitious beliefs regarding their condition.

"I have been attending prayer meetings and all night services at church; I believe that in God's own time, I will have my miracle" (Patient T2-147, Diabetic for 4 years).

"My medication has been prescribed to be taken after meals. When I'm fasting, I don't take my medicines because of potentially unpleasant side effects" (Patient T2-003, Diabetic for 10 years).

\section{Psychosocial barriers}

The impact of diabetes and accompanying side effects from medications affect patients both socially and psychologically. Psychosocial factors such as resignation to fate, fear of medication side effects, perception of health, compliance fatigue and stress and preference for complementary and alternative medicine were cited as reasons for non-compliance among participants.

\section{Resignation to fate}

A common trend in responses recorded during interactions with participants seem to suggest that some of them have become apathetic and resigned to fate. Often, the trigger was a misunderstanding of clinical protocols for management of chronic conditions. Furthermore, it was clear some form of education had been given by healthcare workers to encourage patients to continue taking their medications but participants were left disillusioned instead of feeling motivated.

"I have been counselled by the doctors, nurses and other healthcare staff that this condition will stay with me for the rest of my life so sometimes when I forget to take my medicines, it does not bother me; whatever will be will be" (Patient T2-009, Diabetic for 7 years).

Some participants could not hide their morbid feelings about their condition. For these, the subsequent coping strategy that emanated from such sentiments was a determinant of non-compliance to prescribed medication: 
"As for me, I know that I have this disease until the day I die and there's nothing I can do about it. I'm just living my life the best way I can" (Patient T2-017, Diabetic for 9 years).

\section{Fear of medication side-effects}

Another important determinant of non-compliance was the dread of unpleasant pharmocological effects. Participants were aware of the glucose-lowering effects of the drugs and were occasionally anxious enough to abstain from taking their medication.

"I am a driver... I often skip my medications when I have to drive long distances especially in the mornings because they make me drowsy" (Patient T2-017, Diabetic for 9 years).

Some however feared the perceived effects that could arise from other known and unknown side effects of Metformin

"I skip my medications because ... as a sexually active man, the inability to perform well in bed scares me..." (Patient T2-024, Diabetic for 16 years).

\section{Compliance fatigue and stress}

Patients who were on combination of oral hypoglycaemic drugs and/or drugs for complications often complained of difficulties with medication compliance. The difficulty was largely to do with the effort needed to remember doses and stick to regimen and requirements for food intake. Over time, this leads to fatigue and stressful computations which threaten the sustainability of a compliant lifestyle in long-term diabetics. Here are some extracts from the in-depth interviews:

"Every day, I have to swallow these pills in order to stay alive. I find this rather tiring and boring to be doing the same things over and over" (Patient T2-053, Diabetic for 18 years).

My medicines for hypertension make me urinate a lot" (Patient T2-130, Diabetic for 4 years)

\section{Preference for herbal medication}

Preference for traditional medicine was also a conributing factor to non-compliance among patients as they perceived traditional medicine to be safer and easier to consume than the medicines prescribed by their doctors.

"Sometimes, I prefer herbal concoctions to the pills. Herbal medicine is more natural and safer than the orthodox medicines" (Patient T2-039, Diabetic for 5 years).

\section{Perception of health}

Both favourable and poor perception of health were attributable for non-compliance:

"After taking my medicines for a while, I realised I was feeling better so I thought to myself that there is no need to keep taking them" (Patient T2-008, Diabetic for 7 years). 
"I have stomach ulcer, and sometimes I experience pain after taking these medicines" (Patient T2-018, Daibetic for 5 years).

\section{Socioeconomic status}

Majority of patients interviewed were of low to medium economic status and that was also a contributing factor to non-compliance. Reasons given for non-compliance included medicines running out before next review date, inability to acquire medicines in the local neighborhoods as well as financial constraints when commuting to and from the hospital or clinic site.

"Some of my medications... for cholesterol, are not covered by the NHIS. I am unable to follow my prescription when run out of my supply," (Patient T2-011, diabetic for 5 years).

\section{Barriers to health-seeking behaviour}

A number of reasons given for failure to seek care on time and medication compliance could be classified as health-system related.

\section{Clinic protocols}

A negative perception of clinic protocols persists among a handful of clients. This perception undermines healthcare-seeking behaviour, and may ultimately result in avoidance of medications until complications set-in. For example, patients are required to visit the hospital reception to pick up their folders before going in to see the doctor:

"On clinic days, there is always a long queue where we have to pick up our folders..." (Patient T2-017, Diabetic for 9 years).

\section{Perception of preferential treatment of clients}

Another negative perception has to do with disruptions in consultating room schedules. Some patients complained about long-waiting times and a preferential treatment culture where relatives of hospital staff are not made to join patient queues making other patients more anxious. This can be a threat to honouring appointments for review.

"Sometimes, the doctors attend to other patients who are not in queue because they are friends or close relatives of health staff. That is not fair" (Patient T2-017, Diabetic for 9 years).

\section{Discussion}

This work reports on medication compliance behaviour and contextual determnants of non-compliance in a diabetic clinic in the rapidly urbanizing Bono Region of Ghana. The mixed methods approach was useful for measuring compliance in a comparative manner (14) and allowed the study to triangulate quantitative measures with feedback from defaulting subjects to provide useful data for strengthening healthcare in similar settings. 
The level of medication compliance observed in this study (75.6\%) is higher than what has been reported from patients who visited a teaching hospital in Ghana (38.5\%) (18), in out-patient settings in Dormaa, Ghana (68.5\%) (18) and Nigeria (50.9\%) (11) and among ambulatory patients with type 2 diabetes in a tertiary facility in Nigeria (60.0\%) (20). Generally, medium to high levels of medication compliance are considered to be adequate for optimum glycaemic control (14). Although others have found lower levels of compliance (11), the finding of the present study confirms the position that more effort is needed to understand the determinants of non-compliance and systematically address them.

Religious sentiments that are at variance with clinical advice were a barrier to effective clinical care by undermining medication compliance. There are reports that many Ghanaians, and Africans in general prefer to resort to spiritual healing (whether traditional or Christian) in place of taking medication $(6,8$, 21-23) and this negatively affects compliance. In this study, many respondents suspended taking medication in the exercise of their faith in "spiritual" healing or because they were "fasting" with the hope that God will heal them of their condition in conformity with a similar finding by Atinga et al., (2018).

Other factors that explain the high rate of non-compliance in this study are psychosocial in origin. Some males had a perception that taking their medication could potentially reduce their sexual drive or promote erectile dysfunction. This is consistent with findings from a similar study (18). As the thought of becoming impotent is a source of psychological stress for African men, it has a major influence on medication non-compliance. Indeed, prolonged metformin use has been associated with erectile dysfunction (24).

Compliance fatigue was also reported among patients. In a study with concordant findings (25), an inverse correlation was observed between total number of oral hypoglycaemic drugs taken and compliance: the more pills there were to take, the lower the level of compliance and vice-versa (11). On the contrary, this finding must be interpreted with caution as it may not be replicated among older and highly educated patients (26). Compliance to medication is reported to be associated with socioeconomic status: patients who earned more financially were more adherent while the reverse was observed in those who didn't earn much (6) and indeed there are earlier reports that suggest that majority of noncompliance is from patients with low economic status (7). Here, 84 (52.5\%) out of the 160 participants were self-employed; engaging in farming activities and petty trading which did not fetch much financially. Several complaints were made about not being able to secure transportation fares, or afford medications that are not provided under the NHIS but are prescribed by doctors for other complications and therefore miss doses several times in-between clinic visits. In general insured patients are more likely to report higher compliance than their uninsured counterparts, showing that existing socioeconomic factors cannot be ruled out in strategies to improve medication compliance (11) Although some direct costs of treatment have been eliminated under the NHIS, there is still room for improvement.. The communitybased health planning and services (CHPS) concept offers a strategy to reduce indirect healthcare costs by delivering essential community-based health services in Ghana (27). Feedback from in-depth interviews suggested that interventions that take away transport costs such as shifting patient monitoring by means of point-of-care glucose tests carried out by community health staff who are closer 
to the homes of patients. Making NHIS-listed medicines available at community pharmacies or CHPS compounds, and removal of reported barriers related to the NHIS such as excessive hospital waiting times may further improve medication availability and compliance.

The effect of health system-related disparities on medication compliance has been noted (11). According to the authors, there was a perception among subjects that healthcare workers allocated greater consultation time and better quality medicines to the private clients. Such perceptions may erode the trust of affected patients in the healthcare system, which may consequently affect the patients medication compliance (11). Once identified, there is greater potential for addressing health system related disparities because of higher levels of staff training and responsibility.

\section{Conclusion}

The study shows that misconceptions regarding health and superstition, psychosocial factors related to stress, fate, perception of health, compliance fatigue, socioeconomic factors and barriers to healthseeking behaviour were associated with non-compliance. In addition, barriers to health-seeking behavior such as perceived preferential treatment of card-bearing patients of the National Health Insurance Scheme and long waiting times on clinic days also contributed to non-compliance to medication. It is recommended that counseling sessions should target psychosocial aspects of non-compliance and caregivers should prioritize client relations especially for NHIS card-bearers.

\section{Limitation}

The results of this study should be interpreted for people seeking routine care for diabetes in a clinic setting and may not be representative of other settings. Also, the use of a self-report tool for measuring medication compliance may be a limitation owing to potential for recall bias and tendency of patients to overestimate their compliance behavior.

\section{Declarations}

Conflicts of interest:

none

\section{Acknowledgements:}

The authors are grateful to Dr Issah, Dr. John Ekow Otoo, Director of Public Health and the entire staff of the Regional Directorate of Health Service, Bono Region, Ghana. Special appreciation to Mr. Noah Ayigiri, Mr. Laweh Isaiah Tetteh and staff and patients of Clinic 5 at the Brong-Ahafo Regional Hospital.

\section{References}


1. Stringhini S, Forrester TE, Plange-rhule J, Lambert E V, Viswanathan B, Riesen W, et al. The social patterning of risk factors for noncommunicable diseases in five countries: evidence from the modeling the epidemiologic transition study (METS). BMC Public Health [Internet]. 2016;1-10. Available from: http://dx.doi.org/10.1186/s12889-016-3589-5

2. García-Pérez L-E, Álvarez M, Dilla T, Gil-Guillén V, Orozco-Beltrán D. Adherence to Therapies in Patients with Type 2 Diabetes. Diabetes Ther [Internet]. 2013;4(2):175-94. Available from: http://link.springer.com/10.1007/s13300-013-0034-y

3. Luke A, Bovet P, Forrester TE, Lambert E V., Plange-Rhule J, Schoeller DA, et al. Protocol for the modeling the epidemiologic transition study: A longitudinal observational study of energy balance and change in body weight, diabetes and cardiovascular disease risk. BMC Public Health. 2011;11:1-10.

4. Gudjinu HY, Sarfo B. Risk factors for type 2 diabetes mellitus among out-patients in Ho, the Volta regional capital of Ghana: A case-control study. BMC Res Notes. 2017;10(1):1-10.

5. Islam SMS, Biswas T, Bhuiyan FA, Mustafa K, Islam A. Patients' perspective of disease and medication adherence for type 2 diabetes in an urban area in Bangladesh: a qualitative study. BMC Res Notes. 2017;10(1):1-8.

6. Atinga RA, Yarney L, Gavu NM. Factors influencing long-term medication non-adherence among diabetes and hypertensive patients in Ghana: A qualitative investigation. PLoS One [Internet]. 2018;13(3):1-15. Available from: https://journals.plos.org/plosone/article? id $=10.1371 /$ journal.pone 0193995

7. Sharma T, Kalra J, Dhasmana D, Basera H. Poor adherence to treatment: A major challenge in diabetes. Journal, Indian Acad Clin Med. 2014;15(1):26-9.

8. Kretchy I, Owusu-daaku F, Danquah S. Spiritual and religious beliefs: do they matter in the medication adherence behaviour of hypertensive patients ? Biopsychosoc Med [Internet]. 2013;7(15):1-7. Available from: http://www.bpsmedicine.com/content/7/1/15

9. Quaye EA, Amporful EO, Akweongo P, Aikins MK. Analysis of the Financial Cost of Diabetes Mellitus in Four Cocoa Clinics of Ghana. Value Heal Reg Issues [Internet]. 2015;7:49-53. Available from: http://dx.doi.org/10.1016/j.vhri.2015.08.005

10. Shameena AU, Badiger S, S NK. Medication adherence and health belief model among hypertensive patients attending rural health centres of a tertiary care hospital in South India Medication adherence and health belief model among hypertensive patients attending rural health centres of a. Int $\mathrm{J}$ Community Med Public Heal. 2017;4(4):1159-65.

11. Ajibola SS, Timothy FO. The Influence of National Health Insurance on Medication Adherence Among Outpatient Type 2 Diabetics in Southwest Nigeria. J Patient Exp. 2018;5(2):114-9.

12. Al-Qazaz HK, Hassali MA, Shafie AA, Sulaiman SA, Sundram S, Morisky DE. The eight-item Morisky Medication Adherence Scale MMAS: Translation and validation of the Malaysian version. Diabetes Res Clin Pract. 2010;90(2):216-21. 
13. Ajayi E. Adherence to Antihypertensive Medications and Some of Its Clinical Implications in Patients Seen At a Tertiary Hospital in Nigeria. IOSR J Dent Med Sci. 2013;8(4):36-40.

14. Morisky DE, Ang A, Krousel-Wood M, Ward HJ. Predictive validity of a medication adherence measure in an outpatient setting. J Clin Hypertens. 2008;10(5):348-54.

15. Odegard PS, Gray SL. Barriers to Medication Adherence in Poorly Controlled Diabetes Mellitus. Diabetes Educ. 2008;34(4):692-7.

16. Ramos $\mathrm{Cl}$, Klug JC, Pretto ADB. Compliance With Dietary Recommendations For Users With Type 2 Diabetes. Demetra. 2014;9(2):561-75.

17. Ghana Health Service. Sunyani Regional Hospital Total Number of diabetes cases seen in 2019 [Internet]. [cited 2020 Jun 5]. Available from: https://www.ghanahealthservice.org/hospital.php? hid $=2$

18. Kretchy IA, Owusu-daaku FT, Danquah SA, Asampong E. A psychosocial perspective of medication side effects, experiences, coping approaches and implications for adherence in hypertension management. Clin Hypertens [Internet]. 2015;21(19):1-8. Available from: http://dx.doi.org/10.1186/s40885-015-0028-3

19. Braun V, Clarke V. Using Thematic Analysis in Psycology. Qual Res Psychol. 2006;3(2):1-41.

20. Adisa R, Fakeye TO, Fasanmade A. Medication adherence among ambulatory patients with type 2 diabetes in a tertiary healthcare setting in Southwestern Nigeria. Pharm Pract. 2011;9(2):72-81.

21. Ae-ngibise K, Cooper S, Adiibokah E, Akpalu B, Lund C, Doku V, et al. 'Whether you like it or not people with mental problems are going to go to them ': A qualitative exploration into the widespread use of traditional and faith healers in the provision of Mental Health Care in Ghana. Int Rev Psychiatry. 2010;22(6):558-67.

22. Farnes, C., Beckstrand, R. L. \& Calllister LC. Help-seeking behaviours in childbearing women in Ghana, West Africa. Int Nurs Rev. 2011;58:491-7.

23. Tabi MM, Powell M, Hodnicki D. Use of traditional healers and modern medicine in Ghana. Int Nurs Rev. 2006;53:52-8.

24. Al-Kuraishy H. M. \& Al-gareeb Al. Erectile Dysfunction and Low Sex Drive in Men with Type 2 DM: The Potential Role of Diabetic Pharmacotherapy. J Clin Diagnostic Res. 2016;10(12):21-6.

25. Bruce SP, Acheampong F, Kretchy I. Adherence to oral anti-diabetic drugs among patients attending a Ghanaian teaching hospital. Pharm Pract (Granada). 2015;13(1):1-5.

26. Adisa R, Fakeye TO. Effect of number and type of antidiabetes medications on adherence and glycemia of ambulatory type 2 diabetes patients in southwestern Nigeria. Pharm Pract. 2013;11(3):156-65.

27. Ghana Health Service. Community-based Health Planning and Services [Internet]. [cited 2020 Jun 19]. Available from: https://www.ghanahealthservice.org/chps/category.php? chpscid=98\#: :text=Welcome to Ghana Heath Service,service delivery with the communities. 


\section{Supplementary Files}

This is a list of supplementary files associated with this preprint. Click to download.

- InterviewGuide.docx 